\title{
Effects of ibandronate-hydroxyapatite on resorptive activity of osteoclasts
}

Xiaohui Zhang, Jing Hu, Yunfeng Li, Guozhu Yin, En Luo

State Key Laboratory of Oral Disease, Sichuan University, Chengdu, Sichuan Province, China

Submitted: 17 June 2010

Accepted: 20 August 2010

Arch Med Sci 2011; 7, 1: 53-60

DOI: 10.5114/aoms.2011.20604

Copyright (c) 2011 Termedia \& Banach

\section{Abstract}

Introduction: Bisphosphonates (BPs) can be locally used to improve the osteogenesis around hydroxyapatite (HA) implants. However, there are almost no reports discussing the effects of BPs in the bound state with HA on osteoclasts. Ibandronate is a BP widely used in clinical practice. This study was designed to evaluate the effects of ibandronate combined with HA on the morphology and resorptive activity of osteoclasts.

Material and methods: The HA and ibandronate-HA were prepared. Osteoclasts were isolated from Sprague-Dawley rats and then the cells were cultured with both HA and ibandronate-HA. Then the cell morphology was inspected by inverted phase contrast microscope and transmission electron microscopy observation. The resorptive activity was tested using the dyeing agent seminaphthofluorescein and bone resorption assay.

Results: Compared with the control group, the osteoclasts demonstrated morphological alterations, and the hydrogen ion concentration was significantly lower in the ibandronate-HA group. Areas of the resorption pits formed by the osteoclasts were significantly smaller, the trabecula thickness appeared thicker, and concentration of CTx was also significantly lower in the experimental group. Conclusions: Resorptive activity of osteoclasts cultured with ibandronate-HA was weaker than that of the control group. Ibandronate on HA in the bound state could maintain its action as an inhibitor to osteoclasts.

Key words: hydroxyapatite, bisphosphonate, osteoclast, biomaterial.

\section{Introduction}

Hydroxyapatite (HA) is one of the most commonly used biomaterials for bone scaffolds or implants in surgery and dentistry because of its favourable biocompatibility, bioactivity and osteoconductivity [1]. Bisphosphonates (BPs) are a variety of compounds that prevent the loss of bone mass, clinically used for the treatment of osteoporosis and other disorders of bone metabolism [2]. The BPs, having an affinity for the calcium ions of HA [3], are proven to be a potent inhibitor of bone resorption and an effective accelerator of bone regeneration. The unbound BPs display a common backbone structure of P-C-P. When combined with $\mathrm{HA}$, the line structure of unbound BPs transfers to a six-membered ring structure bound state. It is well known that unbound BPs could be ingested by osteoclasts, activate various effects at the cellular level [4, 5], and then lead to a decrease in the breakdown of bone in vivo. However, there are almost no reports discussing the effects of BPs combined with HA, in the

\author{
Corresponding author: \\ En Luo \\ State Key Laboratory \\ of Oral Disease \\ Sichuan University \\ Renmin South Avenue \\ Chengdu 610041 \\ Sichuan Province, China \\ Phone: +8628 85502334 \\ Fax: +862885582167 \\ E-mail: \\ luoen521125@sina.com
}


bound state, on morphology and resorptive activity of osteoclasts. So it is interesting to study whether $\mathrm{BPs}$ on $\mathrm{HA}$ (in the bound state, a six-membered ring structure) would continue to act as an inhibitor to osteoclasts. And it could provide experimental evidence for BPs modifying HA to improve osteogenesis around $\mathrm{HA}$ in vivo.

Our team has studied the surface properties of HA modified by BPs and its effects on osteoblasts [6-8]. To investigate the effects of BPs combined with $\mathrm{HA}$ on resorptive activity of osteoclasts, we combined HA with ibandronate, one of the nitrogen-containing BPs used widely in clinical practice with a high affinity for HA (calcium ions). Then the morphology and resorptive activity of osteoclasts of Sprague Dawley rats (SD rats) were observed by culturing them with ibandronate-HA and HA for comparison.

\section{Materials and methods}

\section{Material preparation}

The HA bioceramics for bone scaffolds (40.0 MPa compressive strength, $15.5 \mathrm{~mm}$ diameter round shape, $0.5 \mathrm{~mm}$ thickness) were prepared by the Research Center for Nano-Biomaterials, Sichuan University. The ibandronate-HA combination was carried out according to the method of Luo et al. [6-8]. Briefly, the scaffolds were immersed into a solution of $1 \mathrm{mg} / \mathrm{ml}$ ibandronate sodium (SigmaAldrich, USA) for $48 \mathrm{~h}$ at $37^{\circ} \mathrm{C}$ with a $\mathrm{pH}$ of 6.5 to form ibandronate-HA chelate. Then the specimens were cleaned ultrasonically with distilled water for $30 \mathrm{~min}$ and then stored in constant temperature desiccators (as cleaned specimens). The scaffolds without ibandronate were also cleaned and prepared as a control.

\section{In vitro release testing}

Five randomly-selected ibandronate-HA composites were rinsed in $1 \mathrm{ml}$ of eluting agent (adjusted to $\mathrm{pH}$ 7.0). After $24 \mathrm{~h}$, the solutions were collected, and the concentrations of ibandronate were tested by reverse phase liquid chromatography at $220 \mathrm{~nm}$ [9], using a Waters 1525 binary HPLC pump (Waters Co., USA), a Rheodyne (Cotati, CA, USA) model $7725 \mathrm{i}$ injection valve fitted with a $20 \mu \mathrm{l}$ loop, and a Waters 2487 dual $\lambda$ absorbance detector (Waters Co., USA). Ibandronate in the samples was separated on a Phenomenex $\mathrm{C} 18$ column (USA) at $25^{\circ} \mathrm{C}$ using a mixture of acetonitrile and $\mathrm{n}$-amylamine, and the flow rate was $1 \mathrm{ml} / \mathrm{min}$. Such procedures were repeated once a day for 14 days.

\section{Animals and osteoclast culture}

Male rats of SD rat strain (1 day old) were supplied by the Laboratory Animal Center, Sichuan
University. The animal use and care protocol was approved by the Institutional Animal Use and Care Committee of Sichuan University.

Disaggregated osteoclasts were isolated and cultured according to the method of Chambers et al. [10]. Briefly, long bones of the rats were dissected free from soft tissues and sterilized in $75 \%$ ethanol for $5 \mathrm{~min}$. The epiphyses were removed from the bone, which was split longitudinally with a blade. Endosteal surfaces were carefully curetted with a scalpel blade and the bone marrow fragments were collected into culture medium consisting of Dulbecco's minimum essential medium (DMEM) (GIBCO, USA) containing 0.2 g/l glutamine, $100 \mathrm{lU} / \mathrm{ml}$ penicillin, $100 \mathrm{\mu g} / \mathrm{ml}$ streptomycin and $10 \%$ heat-inactivated fetal bovine serum (FBS, Gibco, USA). The bone marrow fragments were pipetted gently to separate cells from the bone fragments. The disaggregated cells were concentrated by centrifugation at $1000 \mathrm{rpm}$ for $10 \mathrm{~min}$ and suspended into culture medium, and then maintained at $37^{\circ} \mathrm{C}$ in an atmosphere of $5 \% \mathrm{CO}_{2}$ for $30 \mathrm{~min}$. The non-adherent cells were washed away with DMEM, and consequently the adherent cells were collected and cultured at $37^{\circ} \mathrm{C}$ in an atmosphere of $5 \% \mathrm{CO}_{2}$. Culture medium was changed every day. The cells were dried and fixed with methanol for $5 \mathrm{~min}$ and stained with $5 \%$ Giemsa solution (Merck, Germany) for 30 min. For tartrate-resistant acid phosphatase (TRAP) assay, an Acid Phosphatase, Leukocyte (TRAP) kit (Sigma, USA) was used according to the manufacturer's instructions. Then the cells were observed under an inverted phase contrast microscope. The TRAPpositive staining multinuclear ( $\geq 3$ nuclei) cells were counted as osteoclasts.

\section{Observation of osteoclasts cultured with scaffolds}

The ibandronate-HA and untreated HA scaffolds were placed in wells of 24-well plates and preincubated overnight in cell culture medium. Then $1 \mathrm{ml}$ of cell suspension $\left(4 \times 10^{3}\right.$ cells $\left./ \mathrm{ml}\right)$ was dripped into each well, so the osteoclasts could precipitate on the surfaces of the scaffolds. The scaffolds and cells were cultured at $37^{\circ} \mathrm{C}$ in an incubator with a $5 \% \mathrm{CO}_{2}$ atmosphere and the medium was changed every other day.

The cells cultured with the ibandronate-HA or pure HA scaffolds were viewed under an IX70-S8F2 inverted phase contrast microscope (IPCM) (Olympus, Japan) and photographed. For transmission electron microscopy (TEM) observation, cells were washed with phosphate buffered solution (PBS) twice and centrifuged at $4000 \mathrm{rpm}$ for $10 \mathrm{~min}$, then fixed with $0.3 \%$ glutaraldehyde for $12 \mathrm{~h}$. Then, the cells were centrifuged at $10000 \mathrm{rpm}$ for $15 \mathrm{~min}$, and fixed with $3 \%$ glutaraldehyde for $2 \mathrm{~h}$. After that, 
the cells were dehydrated in a graded series of ethanol baths, imbedded in araldite, and then underwent ultrathin section prior to being observed under a transmission electron microscope (TEM, JEM-100CX, JEOL, Japan) at $50 \mathrm{kV}$.

\section{Determination of hydrogen ion concentration in osteoclasts}

Osteoclasts of both groups were digested in $0.25 \%$ trypsin with $0.02 \%$ EDTA from the scaffolds and blown into cell suspension. The osteoclasts were treated with $20 \mathrm{mM}$ ammonium chloride for 15 min and washed twice with DMEM free of phenol red or serum, and then placed into a 12-well plate with blood cover slips. After that, the cells were stained with the dyeing agent seminaphthofluorescein (SNAFL), a sort of fluorescent probe that binds specifically with hydrogen ions, and incubated at $37^{\circ} \mathrm{C}$ for $30 \mathrm{~min}$. Twelve osteoclasts out of each well were selected randomly to determine the fluorescence intensity under a confocal microscope with the help of the image analyzing software Laserpix (Bio-Rad, USA). Differences of the data out of each well were analysed using t-test with SPSS10.0 (SPSS, Chicago, IL, USA). Statistical significance was defined as $p<0.05$.

\section{Bone slice resorption assay}

Freshly dissected bovine tibiae and femora free from soft tissues were cleaned and sliced into slices of $5 \mathrm{~mm}$ diameter with the thickness of 10-50 $\mu \mathrm{m}$. Then the slices were cleaned in double distilled water ultrasonically and sterilized with epoxyethane, maintained at $4^{\circ} \mathrm{C}$ in reserve. The bone slices were resterilized under ultraviolet radiation for 30 min every time before use.

Osteoclasts of both groups were digested in $0.25 \%$ trypsin with $0.02 \%$ ethylenediaminetetraacetic acid (EDTA, Sigma, Germany) from the scaffolds and blown into the cell suspension, where density was adjusted to $2 \times 10^{3}$ cells $/ \mathrm{ml}$ after cell counting, for pre-emergency, and seeded into 24-well plates separately, with equal doses of Dulbecco's modified Eagle's medium (DMEM) and one piece of bone slice in each well. The osteoclasts adhere to the surfaces of slices in $30 \mathrm{~min}$. The cells together with the slices were incubated at $37^{\circ} \mathrm{C}, 5 \% \mathrm{CO}_{2}$ atmosphere in a humid incubator. The culture medium was changed every other day. On the 7th day, the bone slices seeded with cells were rinsed with PBS and fixed with 3\% glutaric dialdehyde for $60 \mathrm{~min}$, rinsed again with PBS and then fixed with osmic acid. Afterwards, the slices were dehydrated in descending series of ethanol (50-100\%), soaped with isoamyl acetate for $30 \mathrm{~min}$. Critical point drying and sputter coating were performed before the slices went under the scanning electron microscope
(SEM, JSM-5900LV, JEOL, Japan). A CCD camera was applied to take the picture of the resorption pits and Laserpix software (Bio-Rad, USA) was used to measure the areas of the pits. The 12 largest resorption pits of each bone slice in every well were selected to determine the areas. Mean values of the areas were analysed using t-test with SPSS10.0 (SPSS, Chicago, IL, USA). Statistical significance was defined as $p<0.05$.

The bone microarchitecture at the resorption pits was analysed with a MicroCT $\mu$-CT80 (SCANCO, Switzerland) and then three-dimensional microstructural image data were reconstructed and the trabecular thickness (Tb.Th) of the resorption pits was measured [11]. The data were then analysed using t-test with SPSS10.0 (SPSS, Chicago, IL, USA). Statistical significance was defined as $p<0.05$.

Type I collagen is the main component of bones in mammals. C-telopeptide of type I collagen (CTx) is a specific breakdown product of type I collagen during the process of bone resorption, and usually used as a marker of bone turnover [12]. Levels of CTx in the medium were measured using an enzyme-linked immunosorbent assay (ELISA) kit (Osteometer BioTech, DM) according to the manufacturer's instructions. The concentrations of CTx in each well were measured 3 times and the average values were then analysed using t-test with SPSS10.0 (SPSS, Chicago, IL, USA). Statistical significance was defined as $p<0.05$.

\section{Results}

\section{In vitro release}

During the first 3 days, a sharp decline was observed in the release testing, indicating a rapid ibandronate release from the HA scaffold in the initial period. Then, the release curve went flat from the $4^{\text {th }}$ day on, showing a slow release of ibandronate. The amount of ibandronate was still detectable after 14 days (Figure 1).

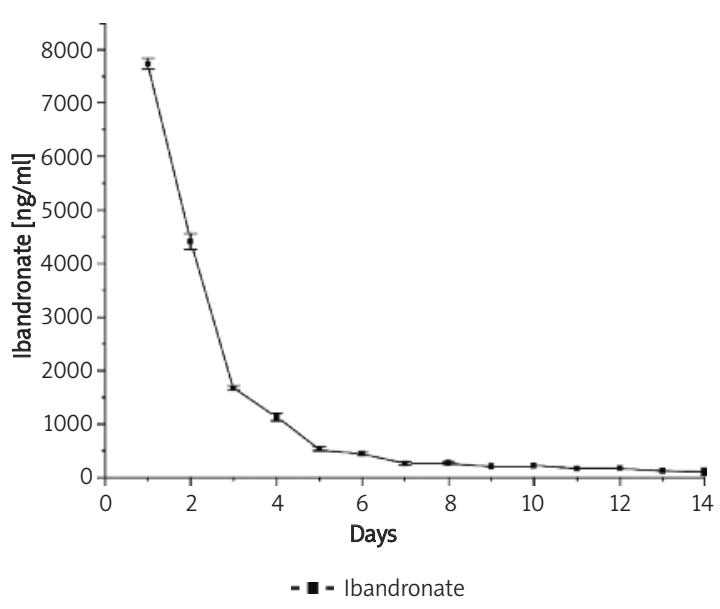

Figure 1. In vitro release of ibandronate release from the HA scaffold 


\section{Osteoclast culture}

In the early stage contained in the cell suspension are osteoclasts and a great amount of other cells, mainly erythrocytes, marrow stroma cells, fibroblasts, etc. The osteoclasts began to adhere in $30 \mathrm{~min}$, while the non-adherent cells were rinsed away. Giant multinucleated osteoclasts with a ruffled border could be clearly distinguished under the inverted phase contrast microscope. The adherent osteoclasts exhibited polymorphism; they had round, oval or irregular shapes (Figure 2. A).

In the Giemsa staining specimen, nuclei of the multinucleated osteoclasts were stained bluish violet, containing 1-2 nucleoli in each nucleus (Figure 2. B). In the TRAP assay, the TRAP positive cytoplasm was dyed ruby red while the TRAP negative nuclei remained unstained (Figure 2. C). 4-20 nuclei could be observed in the multinucleated osteoclasts.

\section{Osteoclasts cultured with scaffolds}

The number of pseudopods decreased markedly after the osteoclasts were cultured with scaffolds in the ibandronate-HA group (Figure 3. A, B). Compared with the control group, osteoclasts cultured with ibandronate-HA were characterized under TEM by condensation of chromatin, reduced number of endoplasmic reticuli, lysosomes and shrinkage of Golgi apparatus. The nucleoli disappeared; the nuclei shrank and became empty inside (Figure 3. C, D).

\section{Hydrogen ion concentration in osteoclasts}

It was observed that relative mean fluorescence intensity (RMFI) of cells in the ibandronate-HA group was significantly lower than that in the control group $(p<0.05)$ (Figure 4$)$. So it could be inferred that the hydrogen ion concentration was lower in the ibandronate-HA group compared with the control group.

\section{Bone slice resorption assay}

Areas of the resorption pits in the bone slices formed by the osteoclasts may reflect the resorption activity. Compared with those of the control group, areas of the pits formed in the ibandronate-HA group were significantly smaller $(P<0.05)$ (Figure 5$)$.

Applying MicroCT and three-dimensional reconstruction, we could clearly determine that Tb.Th at resorption pits in the ibandronate-HA group was thinner than that in the control group $(P<0.05)$ (Figure 6).

The concentration of CTx in the ibandronate-HA group was significantly lower than that in the control group $(P<0.05)$ (Figure 7$)$. It could be inferred that the activity of decomposing type I collagen declined after the osteoclasts were cultured with ibandronate-HA.

\section{Discussion}

In the present study, we investigated the effect of ibandronate in the bound state on resorptive activity of osteoclasts in vitro. The results demonstrated that bound ibandronate is clearly associated with inhibition of osteoclasts. This study might provide experimental evidence for studies regarding the application of BPs modifying HA to improve osteogenesis around HA in vivo.

BPs are synthetic analogues of inorganic pyrophosphate, characterized by a P-C-P group. The affinity for $\mathrm{HA}$ is a property of the P-C-P motif, because it can chelate calcium ions by bidentate coordination through the oxygen atoms of the phosphonate groups [13]. The mechanism of BPs has not been elucidated yet, but there is general agreement that the final target of unbound BPs' action is the osteoclast [14].

After being internalized by osteoclasts, BPs start affecting a series of biochemical processes including both the recruitment and differentiation of osteoclast precursors and the adhesion and activity of mature osteoclasts. Additionally, BPs induce apoptosis of osteoclasts, leading to a decrease in the breakdown of bone. Possible mechanisms of action of BPs are as follows: (a) inhibition of osteoclast recruitment to the bone surface; (b) inhibition of osteoclast activity on the bone surface; (c) shortening of the osteoclast life span; and (d) alteration of bone mineral [15]. A consequence of the first three effects could be a reduced number of osteoclasts.

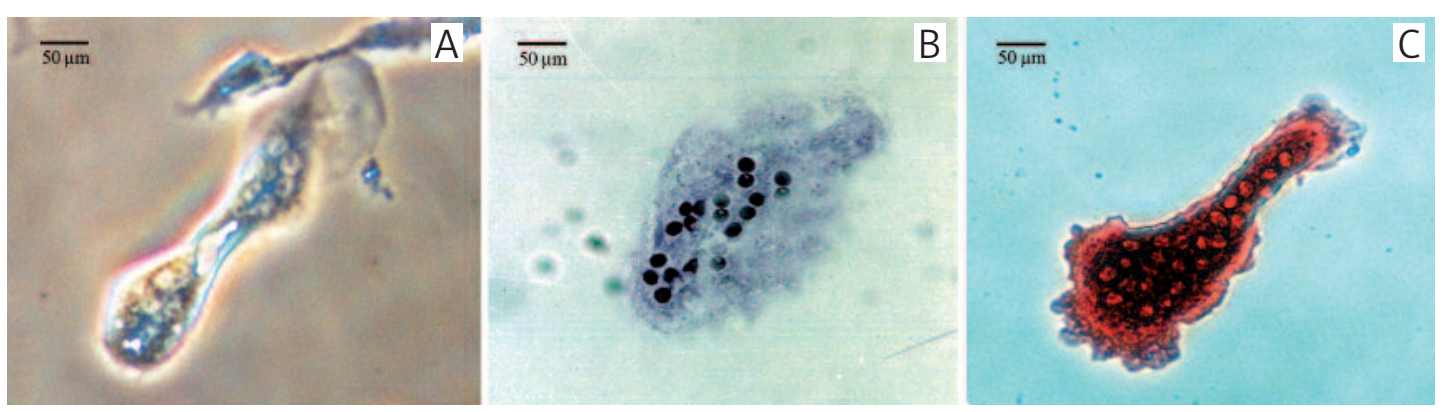

Figure 2. A - Multinucleated osteoclast observed under IPCM. B - Giemsa staining. C - TRAP assay 

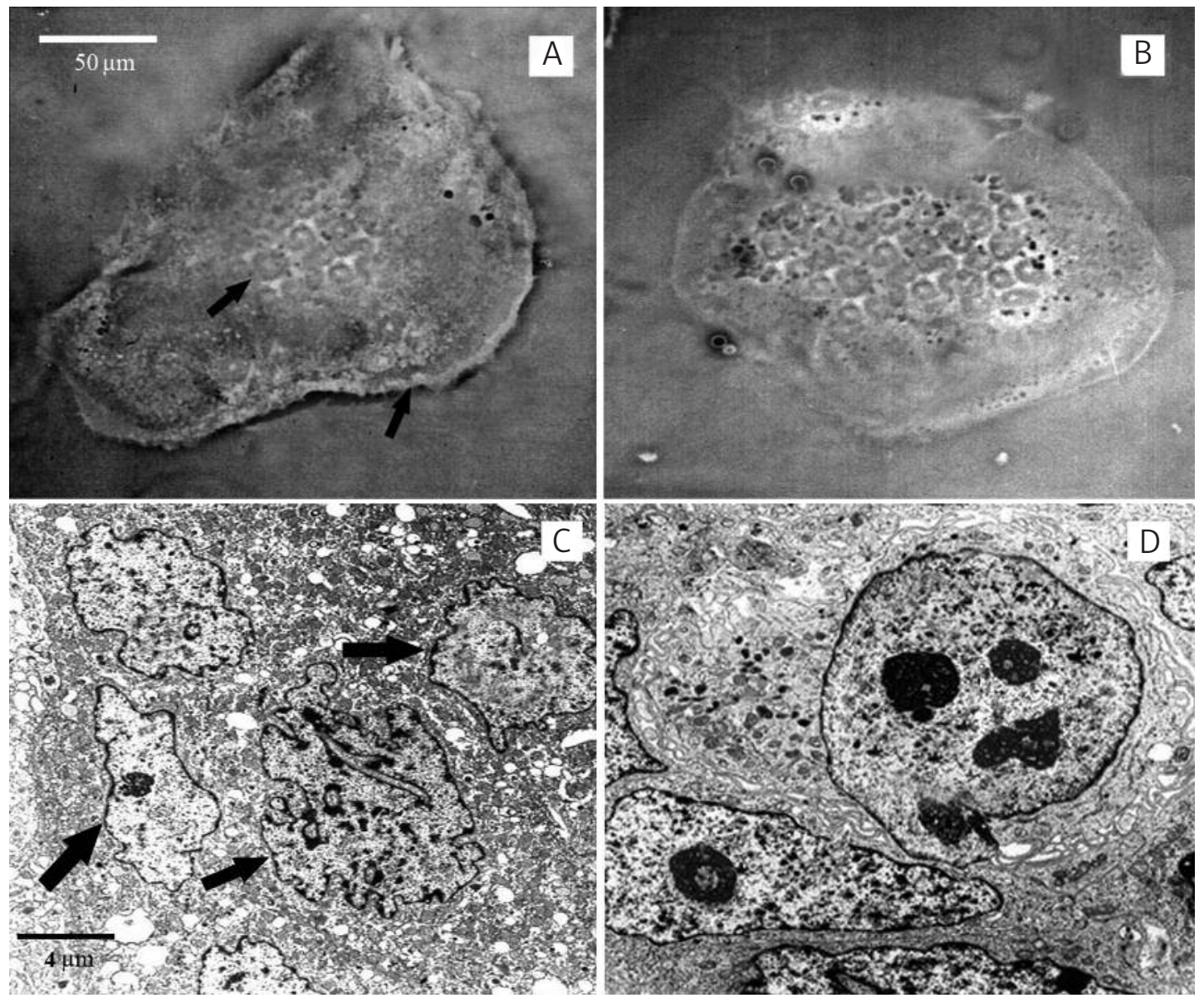

Figure 3. Osteoclasts of both groups after being cultured with scaffolds through IPCM and TEM. The pseudopods of osteoclasts in ibandronate-HA groups decreased markedly with shrunk or empty nuclei (as the arrows indicate) (A), while nuclei of osteoclasts cultured with HA have a basically normal shape (B). Osteoclasts cultured with ibandronateHA had collapsed nuclei and shrunken organelles (as the arrows indicate) (C). Osteoclasts in the control group had basically normal organelles (D)

In this in vitro study, the characteristic morphological features of ibandronate-treated osteoclasts were the lack of ruffled borders, shrunken nuclei and decreased size of organelles. A possible explanation is that ibandronate was converted intracellularly to a nonfunctional analogue of ATP that competed with ATP in the cellular energy metabolism. The osteoclasts initiated apoptosis and died [16]. During apoptosis, osteoclasts lost their cytoskeletal integrity and ruffled borders, nuclei shrank, and osteoclasts became inactive.

In osteoclastic bone resorption, $\mathrm{H}^{+}$is produced from $\mathrm{CO}_{2}$ and $\mathrm{H}_{2} \mathrm{O}$ by carbonic anhydrase II intracellularly and secreted extracellularly by $\mathrm{H}^{+}$-ATPase in the membrane of the ruffled border [17]. Accumulation of large amounts of protons in the resorption lacuna causes acidification of the resorption pit and initiates inorganic mineral dissolution. David et al. found that tiludronate, a non-nitrogenous BP, was a potent inhibitor of proton-pumping V-ATPase [18]. Clodronate, etidro- nate, pamidronate and alendronate could inhibit vacuolar acidification in some form of metabolic inhibition, which did not appear to be direct inhibition of ATPase [19]. Our results demonstrated that bound ibandronate could lead to a decline of intracellular proton concentration. This is consistent with the previous findings.

Effects of immobilized BPs on bone defect repair or osteogenesis around HA-coated implants have been investigated in some in vivo studies. The results demonstrated that BPs could accelerate bone defect repair and improved osteogenesis around HA [20]. But few mechanisms have been described. Our findings could give a possible explanation in terms of bone remodelling. After $\mathrm{HA}$ is implanted into the defects, the bone tissue will undergo a process of remodelling according to the basic multicellular unit theory [21]. It is expected that the osteogenic activity will gain the advantage in the formation phase of bone remodelling for the successful 

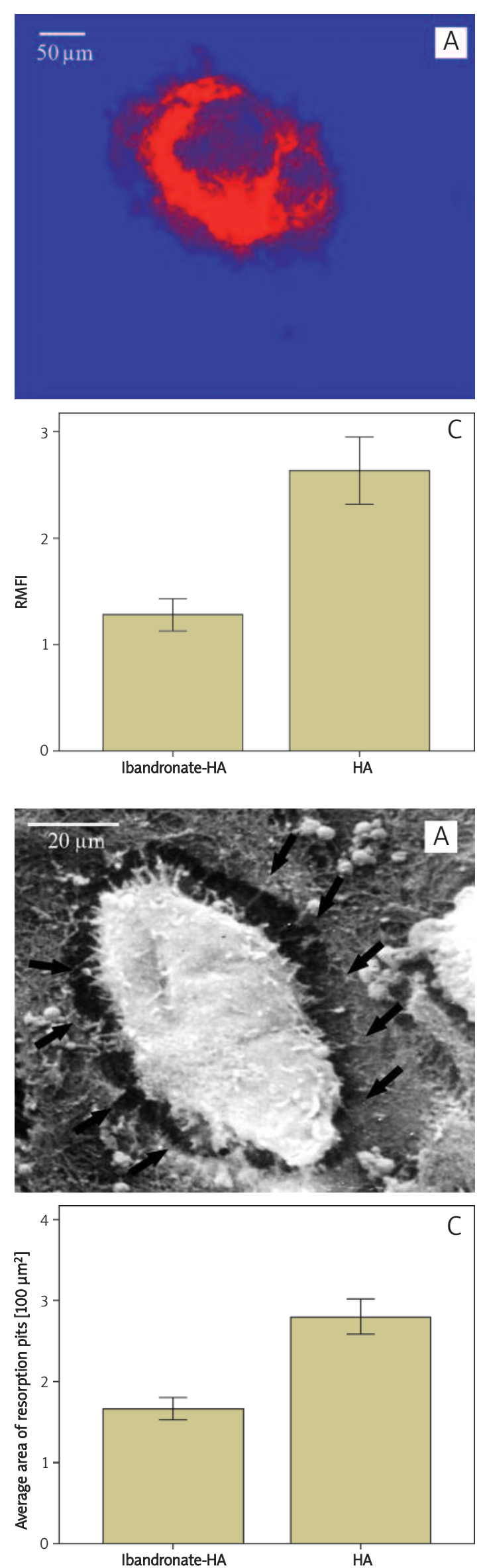

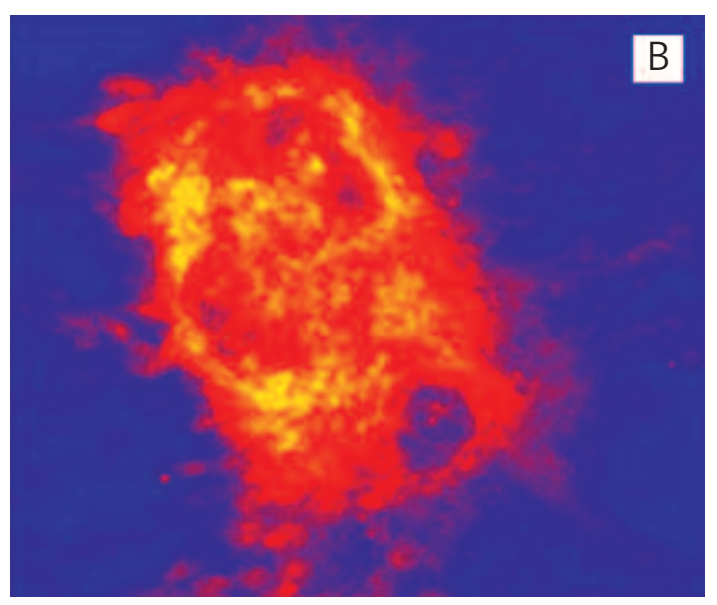

Figure 4. Osteoclasts in both groups stained by SNAFL through confocal microscope. Hydrogen ion concentration in the ibandronate-HA group was lower, and osteoclasts stained by SNAFL had weaker fluorescence intensity (A) compared with the control group (B)

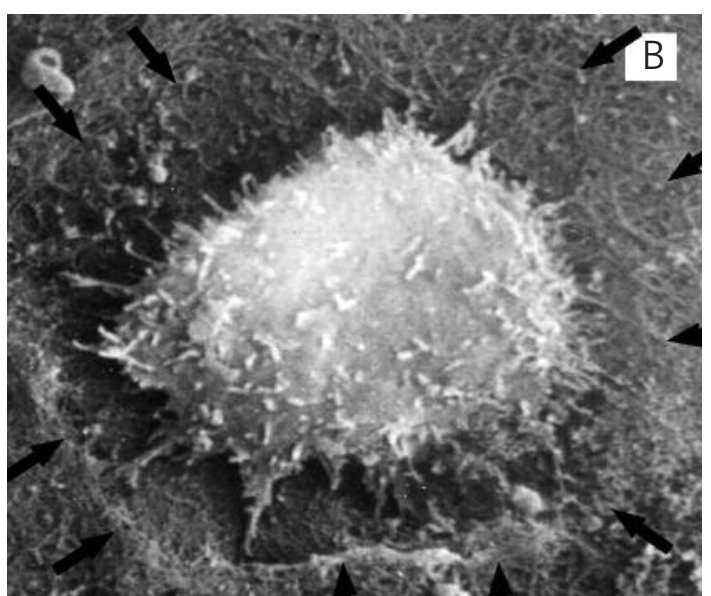

Figure 5. Osteoclasts in ibandronate-HA group formed smaller resorption pits (as the arrows indicate) (A) compared with the control group (B) 

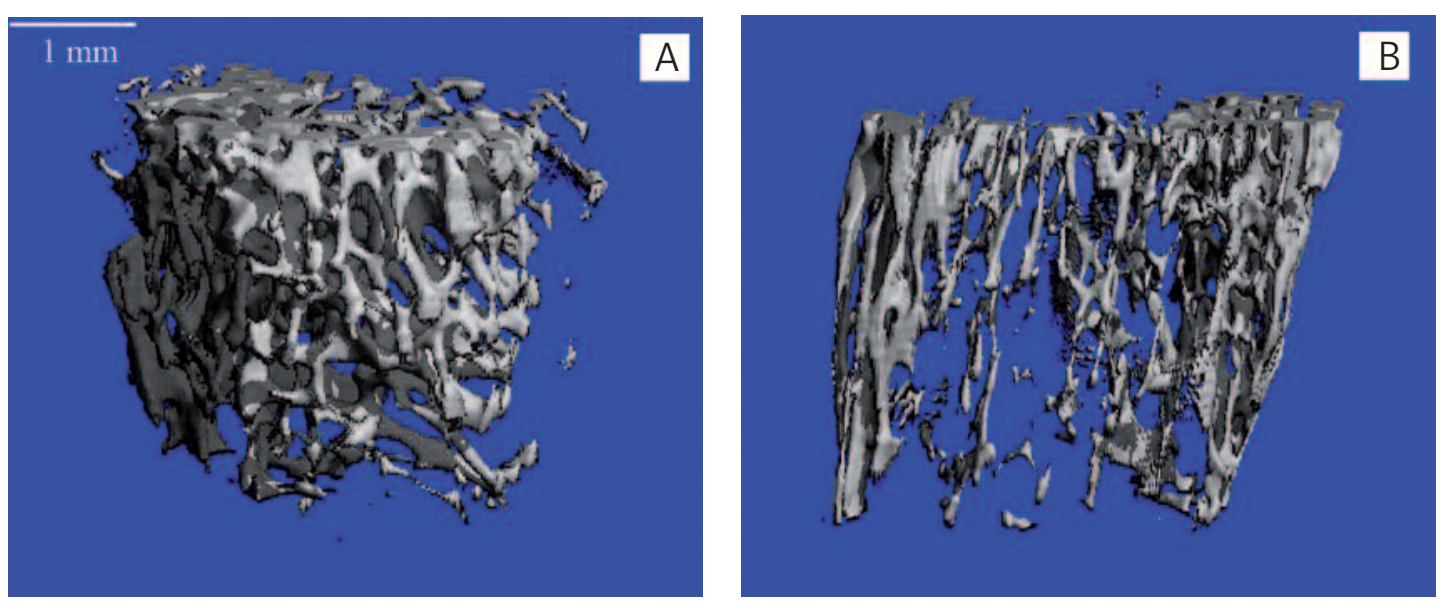

Figure 6. Resorption pits in 3-D reconstruction of microCT. Tb.Th at resorption pit in the ibandronateHA group (A) was significantly thicker than that in the control group (B)

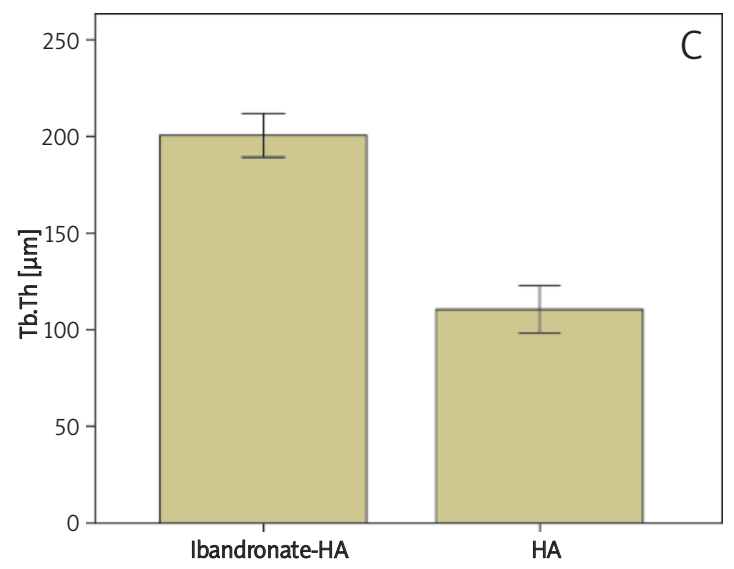

restoration of the bone defect. However, osteoclasts remain hyperactive in the formation phase due to injury, local infection, undesirable stress and poor biocompatibility of biomaterials, which may lead to peri-HA osteolysis and subsequent loosening [22]. Therefore, when the activity of osteoclasts at the HA implant site is moderately inhibited by BPs in the bone formation phase, both the osteogenesis around the implants and the biointegration between bones and implants are improved. In clinical practice, if HA scaffolds or HA-coated implants are used in sites involved by infection, undesirable stress, or osteoporosis [23], pre-treatment with BPs might be a novel and effective option. Further study is required to more precisely define the proper types of BPs, the release dose, and the methods of combining BPs with $\mathrm{HA}$.

In this study, rat osteoclasts were cultured with ibandronate-HA and untreated HA scaffolds. Results showed that, compared with those cultured with untreated $\mathrm{HA}$, ibandronate-HA could lead to osteoclasts' morphological changes and weaker resorptive activity. Evidence was obtained indicating that ibandronate on HA in the bound state could continue to act as an inhibitor to osteoclasts, and

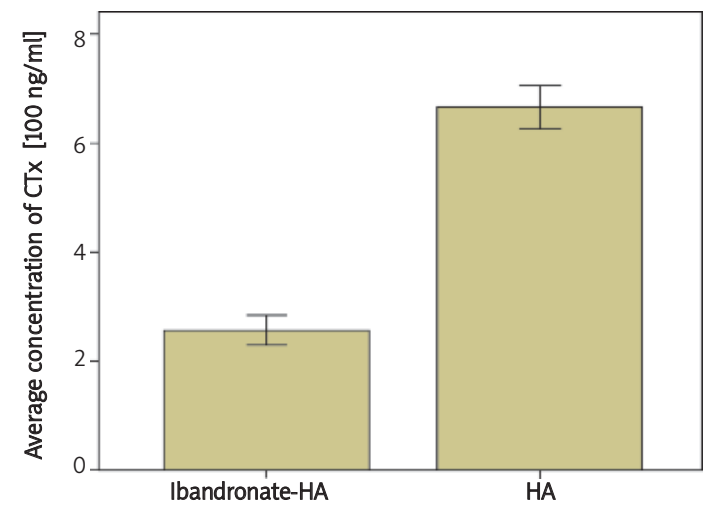

Figure 7. Concentration of CTx in the ibandronateHA group was significantly lower than that in the control group $(P<0.05)$

further experiments should be done to confirm this conclusion.

\section{Acknowledgments}

This study was supported by grants from the financial support of the National Science Foundation of China (30700950 and 30973346), the Doctoral Specialty Foundation of High School (20070610061). 


\section{References}

1. He G, Hu J, Wei SC, Li JH, Liang XH, Luo E. Surface modification of titanium by nano-TiO2/HA bioceramic coating. Applied Surface Science 2008; 255: 442-5.

2. Makras P, Hamdy NAT, Zwinderman AH. Bisphosphonate dose and incidence of fractures in postmenopausal osteoporosis. Bone 2009; 44: 766-71.

3. Cukrowski I, Popovic L, Barnard W, Paul SO, van Rooyen $\mathrm{PH}$, Liles DC. Modeling and spectroscopic studies of bisphosphonate-bone interactions. The Raman, NMR and crystallographic investigations of Ca-HEDP complexes. Bone 2007; 41: 668-78.

4. Rogers MJ, Gordon S, Benford HL, et al. Cellular and molecular mechanisms of action of bisphosphonates. Cancer 2000; 88: 2961-78.

5. Kajiwara H, Yamaza T, Yoshinari $M$, et al. The bisphosphonate pamidronate on the surface of titanium stimulates bone formation around tibial implants in rats. Biomaterials 2005; 26: 581-7.

6. Luo E, Wei SC, He G, Xiao L, Zhao Q. The bisphosphonate clodronate modifying hydroxyapatite bioceramics for bone scaffold. J. Wuhan Univ Technol 2005; 20: 98-101.

7. Luo E, Liu X, Wei SC, Cai XX, Hu J. Osteoblast adhesion to clodronate-hydroxyapatite composite. Applied Surface Science 2008; 255: 308-11.

8. Luo E, Cui J, Gao Y, Lin YF, Zhu SS, Hu J. Effect of pamidronate on protein adsorption and osteoblast adhesion to hydroxyapatite bioceramics scaffold. Key Engineering Materials 2007; 330: 885-8.

9. Xie Z, Jiang Y, Zhang DQ. Simple analysis of four bisphosphonates simultaneously by reverse phase liquid chromatography using $\mathrm{n}$-amylamine as volatile ion-pairing agent. J Chromatogr A 2006; 1104: 173-8.

10. Chambers TJ, Magnus CJ. Calcitonin alters behaviour of isolated osteoclasts. J Pathol 1982; 136: 27-39.

11. Swain VM, Xue J. State of the Art of Micro-CT Applications in Dental Research. International Journal of Oral Science 2009; 4: 177-88.

12. Garnero P, Schott AM, Prockop D, Chevrel G. Bone turnover and type I collagen C-telopeptide isomerization in adult osteogenesis imperfecta: Associations with collagen gene mutations. Bone 2009; 44: 461-6.

13. Rogers MJ, Watts DJ, Russell RG. Overview of bisphosphonates. Cancer 1997; 80: 1652-60.

14. Russell RGG, Rogers MJ. Bisphosphonates: from the laboratory to the clinic and back again. Bone 1999; 25: 97-106.

15. Fleisch H. Bisphosphonates: mechanisms of action. Endocr Rev 1998; 19: 80-100.

16. Frith JC, Monkkonen J, Blackburn GM, Russell RG, Rogers MJ. Clodronate and liposome-encapsulated clodronate are metabolized to a toxic ATP analog, adenosine 5'-(beta, gamma-dichloromethylene) triphosphate, by mammalian cells in vitro. J Bone Miner Res 1997; 12: 1358-67.

17. Riihonen R, Supuran CT, Parkkila S, Pastorekova S, Väänänen HK, Laitala-Leinonen T. Membrane-bound carbonic anhydrases in osteoclasts. Bone 2007; 40: 1021-31.

18. David P, Nguyen H, Barbier A, Baron R. The bisphosphonate tiludronate is a potent inhibitor of the osteoclast vacuolar H(+)-ATPase. J Bone Miner Res 1996; 11: 1498-507.

19. Zimolo Z, Wesolowski G, Rodan GA. Acid extrusion is induced by osteoclast attachment to bone. Inhibition by alendronate and calcitonin. J Clin Invest 1995; 96: 2277-83.

20. Yoshinari M, Oda Y, Ueki H, Yokose S. Immobilization of bisphosphonates on surface modified titanium. Biomaterials 2001; 22: 709-15.
21. Epker BN, Frost HM. Correlation of bone resorption and formation with the physical behavior of loaded bone. J Dent Res 1965; 44: 33-41.

22. Wang ML, Sharkey PF, Tuan RS. Particle bioreactivity and wear-mediated osteolysis. J Arthroplasty 2004; 19: 1028-38.

23. Kandil ME, Mourad A, El Hamshary A. Evaluation of bone mineral density and bone turnover markers in Egyptian children with juvenile rheumatoid arthritis. Arch Med Sci 2009; 5: 434-42. 